\title{
TARANIS XGRE and IDEE detection capability of terrestrial gamma-ray flashes and associated electron beams
}

\author{
David Sarria $^{1}$, Francois Lebrun ${ }^{1,2}$, Pierre-Louis Blelly ${ }^{3,4}$, Remi Chipaux ${ }^{5}$, Philippe Laurent ${ }^{1,2}$, \\ Jean-Andre Sauvaud ${ }^{3,4}$, Lubomir Prech $^{6}$, Pierre Devoto ${ }^{3,4}$, Damien Pailot ${ }^{1}$, Jean-Pierre Baronick ${ }^{1}$, and \\ Miles Lindsey-Clark ${ }^{1}$ \\ ${ }^{1}$ APC, AstroParticule et Cosmologie, Universite Paris Diderot, CNRS/IN2P3, CEA/DRF/IRFU, Observatoire de Paris, \\ Sorbonne Paris Cite, 10 rue Alice Domont et Leonie Duquet, 75205 Paris CEDEX 13, France \\ ${ }^{2}$ CEA/DRF/IRFU/Sap, Bat. 709, Orme des Merisiers, CEA-Saclay, 91191 Gif-sur-Yvette CEDEX, France \\ ${ }^{3}$ Universite de Toulouse, UPS-OMP, IRAP, Toulouse, France \\ ${ }^{4}$ CNRS, IRAP, 9 Av. colonel Roche, Toulouse, France \\ ${ }^{5}$ CEA/DRF/IRFU/SEDI, CEA-Saclay, 91191 Gif-sur-Yvette CEDEX, France \\ ${ }^{6}$ Faculty of Mathematics and Physics, Charles University, Prague, Czech Republic
}

Correspondence to: David Sarria (david.sarria.89@gmail.com)

Received: 31 January 2017 - Discussion started: 24 February 2017

Revised: 11 June 2017 - Accepted: 14 June 2017 - Published: 13 July 2017

\begin{abstract}
With a launch expected in 2018, the TARANIS microsatellite is dedicated to the study of transient phenomena observed in association with thunderstorms. On board the spacecraft, XGRE and IDEE are two instruments dedicated to studying terrestrial gamma-ray flashes (TGFs) and associated terrestrial electron beams (TEBs). XGRE can detect electrons (energy range: 1 to $10 \mathrm{MeV}$ ) and $\mathrm{X}$ - and gammarays (energy range: $20 \mathrm{keV}$ to $10 \mathrm{MeV}$ ) with a very high counting capability (about 10 million counts per second) and the ability to discriminate one type of particle from another. The IDEE instrument is focused on electrons in the $80 \mathrm{keV}$ to $4 \mathrm{MeV}$ energy range, with the ability to estimate their pitch angles.

Monte Carlo simulations of the TARANIS instruments, using a preliminary model of the spacecraft, allow sensitive area estimates for both instruments. This leads to an averaged effective area of $425 \mathrm{~cm}^{2}$ for XGRE, used to detect Xand gamma-rays from TGFs, and the combination of XGRE and IDEE gives an average effective area of $255 \mathrm{~cm}^{2}$ which can be used to detect electrons/positrons from TEBs. We then compare these performances to RHESSI, AGILE and Fermi GBM, using data extracted from literature for the TGF case and with the help of Monte Carlo simulations of their mass models for the TEB case.
\end{abstract}

Combining this data with the help of the MC-PEPTITA Monte Carlo simulations of TGF propagation in the atmosphere, we build a self-consistent model of the TGF and TEB detection rates of RHESSI, AGILE and Fermi. It can then be used to estimate that TARANIS should detect about 200 TGFs $\mathrm{yr}^{-1}$ and $25 \mathrm{TEBs}^{-1} \mathrm{r}^{-1}$.

\section{Introduction}

Terrestrial gamma-ray flashes (TGFs) are short $(\sim 20 \mu \mathrm{s}$ to $\sim 1 \mathrm{~ms}$ ) $\mathrm{X}$ - and gamma-ray emissions associated with lightning and mostly detected from space. Together with transient luminous events for the optical part (see Surkov and Hayakawa, 2012, for a comprehensive review), they play an important role in understanding the coupling between magnetosphere-ionosphere-atmosphere. A comprehensive review of TGFs and related studies, called the High Energy Atmospheric Physics, is provided by Dwyer et al. (2012). Detections of TGFs from space were first presented by Fishman et al. (1994) using data from the Burst And Transient Source Experiment (BATSE) on board NASA's Compton Gamma-Ray Observatory. In the subsequent years, TGFs were also detected from space by other satellites: the Reuven Ramaty High Energy Solar Spectroscopic Im- 
ager (RHESSI) (Smith et al., 2005), the Astro-rivelatore Gamma a Immagini Leggero (AGILE) (Marisaldi et al., 2014) and the Fermi space telescope (Briggs et al., 2010). Very recently, TGF events were also found in the BeppoSAX data archive (Ursi et al., 2017).

A careful analysis of BATSE, RHESSI and Fermi-GBM data permitted the identification of some longer events with durations more than $1 \mathrm{~ms}$ (Smith et al., 2006; Dwyer et al., 2008; Cohen et al., 2010; Briggs et al., 2011). These events were not directly due to the detection of gamma rays, but to secondary electrons and positrons produced by the TGF, and were called terrestrial electron beams (TEBs). Contrary to gamma rays, the charged particles are beamed by the magnetic field of the Earth, can travel thousands of kilometres from one hemisphere to the other and may be detected in unusual locations for TGFs; e.g. the Fermi 091214 event was detected above the Egyptian desert (Briggs et al., 2011; Sarria et al., 2016). These electrons/positrons can then be trapped by the geomagnetic field and they may provide a significant source of high-energy $(>1 \mathrm{MeV})$ particles to the radiation belts. The impact of TEBs on radiation belts still needs to be quantified.

In the near future, two missions are planned with the primary objective of TGF detection: ASIM and TARANIS. The Atmosphere-Space Interaction Monitor (ASIM) is an European Space Agency (ESA) project with scientific leadership from the Technical University of Denmark (DTU) (Neubert et al., 2006). It will employ two X-and gamma-ray detectors (MXGS-LED and MXGS-HED) coupled with optical sensors (MMIA). It will be docked on the International Space Station (ISS) in the course of 2017. The Tool for the Analysis of RAdiation from lightNIng and Sprites (TARANIS) is a microsatellite belonging the French Space Agency (CNES) which will be dedicated to the study of transient events related to thunderstorm activity (Lefeuvre et al., 2009) and will be launched in 2018. All instruments on board the TARANIS spacecraft collaborate for the transient event study. Upon an alert from one instrument, all instruments can record data prior to, during and after the trigger. Two instruments have been specifically designed to study TGFs and TEBs: the instrument for X-Gamma-Ray and Relativistic Electrons (XGRE) and the Instrument for Detection of Energetic Electrons (IDEE). XGRE and IDEE are two of the four instruments that have the ability to trigger all the on-board instruments.

These two instruments will be presented in Sect. 2. In Sect. 3 we will make a comparison of the performances of XGRE and IDEE with those of RHESSI, Fermi-GBM and AGILE-MCAL in the context of TGFs. Finally in Sect. 4 we build a self-consistent picture to account for the detection rates of TGF and TEB seen by the satellites flying today in order to estimate the future detection rates of TARANIS.

\section{The TARANIS XGRE and IDEE instruments}

\subsection{The XGRE instrument}

XGRE can detect photons in the $[20 \mathrm{keV}-10 \mathrm{MeV}]$ energy range and electrons in the $[1 \mathrm{MeV}-10 \mathrm{MeV}]$ energy range. There are major differences when detecting photons (from TGF) and electrons or positrons (from TEB). Photons in this energy range always have a probability of not interacting with a given material, whereas an electron crossing a given material always deposits energy in it. A significant increase in photon energy always implies a significant increase in the average energy deposit on the detection material, allowing a proper estimate of the incident photon energy spectrum. The XGRE instrument also has the ability to trigger the other instruments of the TARANIS payload.

In general, the energy of an incident electron is difficult to estimate properly. Using several layers of detectors greatly helps but there remain uncertainties due to the detector's environment. Positrons will behave very similarly to electrons, with the addition that they will always annihilate into two $511 \mathrm{keV}$ photons once they have lost most of their kinetic energy.

The XGRE instrument is presented in Fig. 1. Figure 1a shows its position on the TARANIS satellite (highlighted in red). XGRE is composed of three sensors that are tilted by $20^{\circ}$ with regard to the payload baseplate. The relative counts of the three sensors allow for an estimate of the direction of the gamma-ray flux for bright events (more than 100 counts). Simulations show that the angular resolution is about $38^{\circ}$ for a TGF that produces 100 counts, $27^{\circ}$ for 200 counts and $18^{\circ}$ for 400 counts. However these estimations have yet to be confirmed using measurements of the satellite calibration campaign (expected in 2018). Each sensor contains four detection units, as presented in Fig. 1b. Each unit has one $8.7 \mathrm{~mm}$-thick lanthanum bromide crystal $\left(\mathrm{LaBr}_{3}\right)$ scintillator, surrounded by two $5 \mathrm{~mm}$-thick plastic scintillators, as shown in Fig. 1c, and the three scintillators are connected to two multi-anode photo-multipliers. This sandwich design allows the identification of the triggering photon or charged particle.

The plastic scintillators have a low effective atomic number $(Z \approx 12)$ and a low density $\left(1.03 \mathrm{~g} \mathrm{~cm}^{-3}\right)$; therefore gamma rays have a small probability of interacting with it and/or depositing all of their energy. On the other hand, gamma rays have a much higher probability of interacting with the $\mathrm{LaBr}_{3}$ due to their high effective atomic number $(Z=46.9)$, five-times-higher density $\left(5.08 \mathrm{~g} \mathrm{~cm}^{-3}\right)$ and larger thickness. The three scintillators are sensitive to charged particles. If a significant amount of energy is deposited in the $\mathrm{LaBr}_{3}$ only, it will probably be due to a gamma ray. If some energy is deposited in a plastic scintillator only, it will likely be due to an electron with energy below $1.2 \mathrm{MeV}$. If energy is deposited in one (or two) plastic(s) and in a $\mathrm{LaBr}_{3}$ crystal, it will likely be due to a higher energy electron (above 1.2 MeV). 
(a)

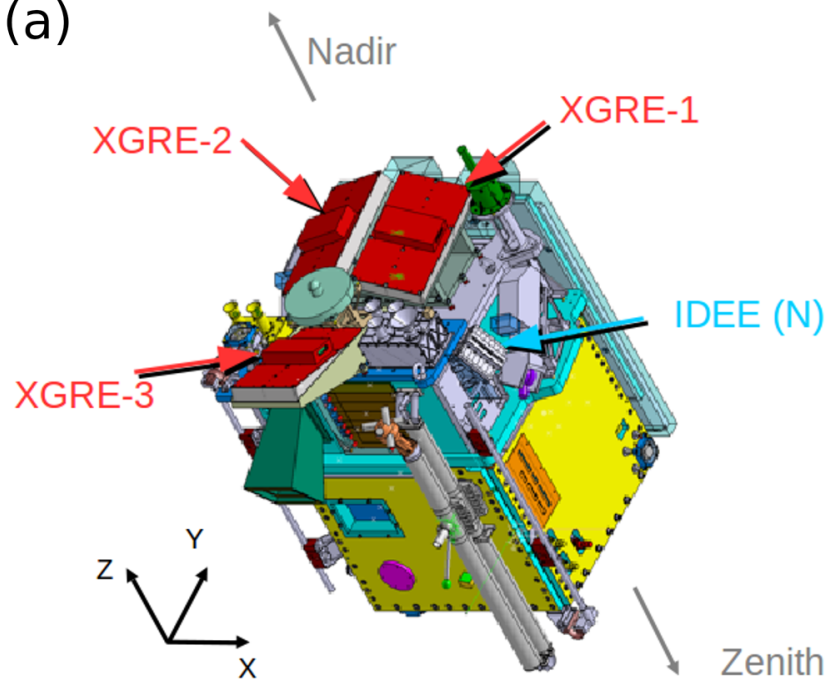

(b)

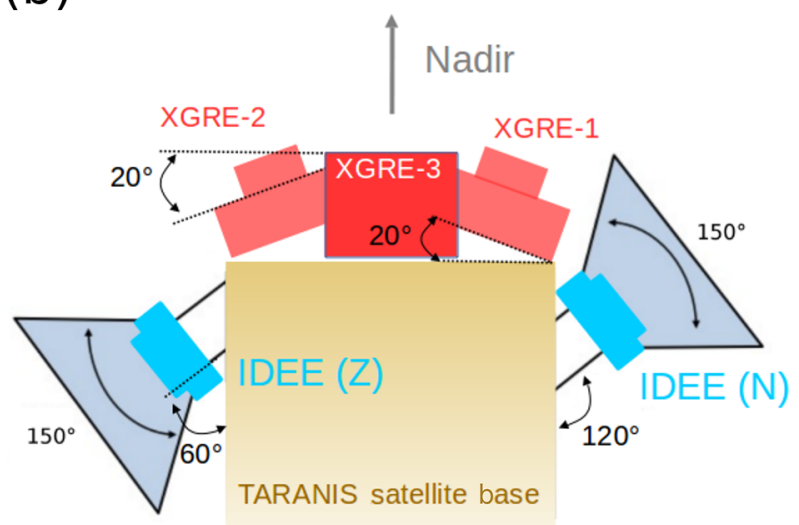

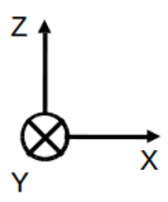

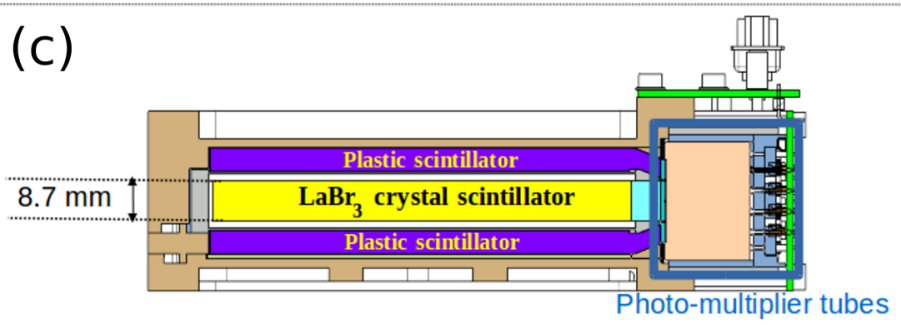

(d)

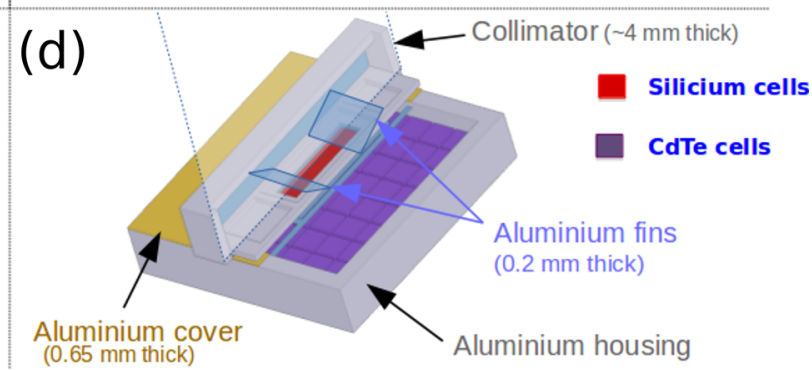

Figure 1. (a) 3-D view of the TARANIS spacecraft. The position of the three XGRE sensors are indicated, as well as one of the two IDEE detectors (the other is hidden). (b) Schematic side views of the TARANIS spacecraft. The three XGRE sensors are highlighted in red and the two IDEE detectors are highlighted in cyan. The relative positioning and orientation of the instruments are accurately represented. For more clarity, the relative scale of the two IDEE detectors is slightly bigger than in reality. (c) Cross-section view of a XGRE detector unit, highlighting the sandwich design of plastic/LaBr 3 scintillators. (d) Partial cross-section view of one IDEE detector's head. The position of the sensitive cells used to detect electrons (silicon and CdTe) are highlighted.

The effective area of XGRE for detecting gamma rays could be determined using the GEANT4 full mass model of the instrument and satellite. GEANT4 is a toolkit developed by a international collaboration led by CERN to simulate the propagation of particles though matter (Agostinelli et al., 2003; Allison et al., 2006). It is an essential tool for simulating high-energy particle detectors and to estimate their performance.

Two side views of the GEANT4 mass model of XGRE are presented in Fig. 1a. This mass model will be refined in the coming years, using results of calibration campaigns.

To determine the response of the detector to X-rays and gamma rays, we drawn 150 mono-energetic beams of $20 \times$ $10^{6}$ photons, each with a different energy between $20 \mathrm{keV}$ and $20 \mathrm{MeV}$. The particles are drawn from the direction of nadir, towards the satellite. Indeed, the attitude of the satellite is such that the detector will always point towards the Earth (nadir).
Any particle that deposits energy above the electronic trigger is considered to be detected, i.e. above $300 \mathrm{keV}$ on a plastic scintillator and/or above $20 \mathrm{keV}$ in a $\mathrm{LaBr}_{3}$ crystal.

Figure 2a shows the computed effective area of XGRE for gamma rays, using $\mathrm{LaBr}_{3}$ (black curve). The effective area of XGRE is maximal at $E_{\max }(\sim 125 \mathrm{keV})$ with an effective area above $836 \mathrm{~cm}^{2}$. Below $E_{\max }$, the effective area decreases as weaker $\mathrm{X}$-rays are more easily absorbed by materials surrounding the crystal (e.g. plastic scintillators, aluminium housing, hoods). The effective area is negligible below $20 \mathrm{keV}$ by design. Above $E_{\max }$, the effective area decreases to $\approx 190 \mathrm{~cm}^{2}$ at $1.5 \mathrm{MeV}$. For higher energies, the pair production probability (by interaction with the detector or the surrounding material) becomes higher, increasing the effective area, which reaches $\approx 230 \mathrm{~cm}^{2}$ at $20 \mathrm{MeV}$.

In Appendix A, we describe how we can calculate an average effective area to obtain a unique value associated to a detector for detecting TGF or TEB. For the average effective area of XGRE, the calculation gives $\sigma_{\mathrm{XG}}^{\mathrm{TGF}} \approx 425 \mathrm{~cm}^{2}$ 

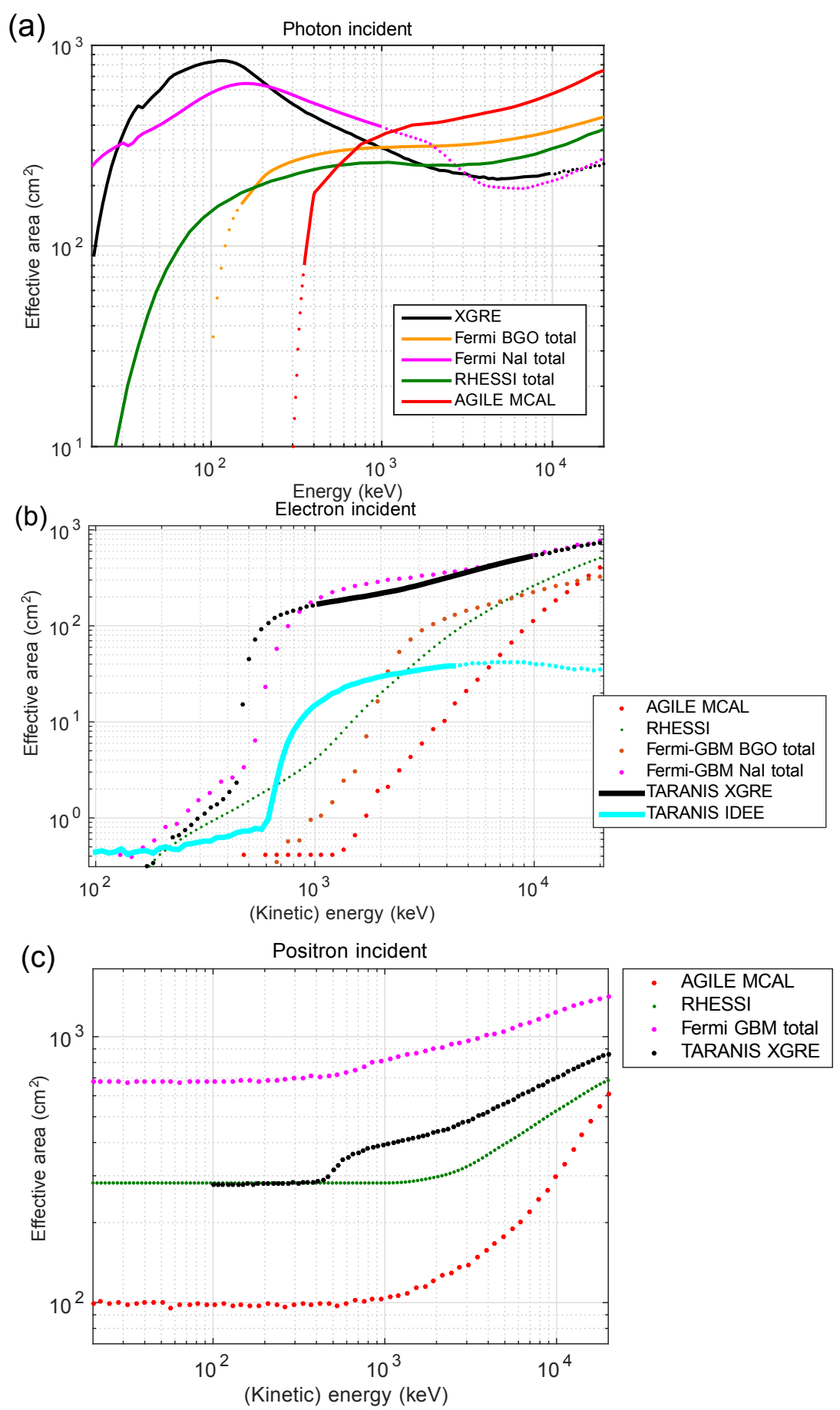

Figure 2. (a) Effective area for X-rays and gamma rays vs. energy for the considered detectors. (b) Effective area for electrons vs. energy for the considered detectors. (c) Effective area for positrons vs. energy for the considered detectors. A full line indicates that the spectroscopy is possible at the given energy, and a dot line indicates that it is not. TARANIS XGRE and IDEE data come from GEANT4 simulations performed for this work. Concerning photon response (a), the data for Fermi-GBM were obtained by averaging about 100 response matrices generated from publicly available tools provided by the Fermi-GBM collaboration. For RHESSI, the data were extracted from the response matrix provided by D. Smith. For AGILE-MCAL, this data are reproduced from literature. Concerning the response to electrons and positrons (b and $\mathbf{c}$ ), these results are obtained from GEANT4 and GEANT3 simulations using the mass models of the spacecrafts (or an approximative version for Fermi). Complete references are presented in the code and data availability sections. 
for a TGF source located at nadir. If the TGF source is offset from nadir, the value of $\sigma_{\mathrm{XG}}^{\mathrm{TGF}}$ goes down to $401 \mathrm{~cm}^{2}$ $(-5.6 \%)$ for a $20^{\circ}$ angle, $354 \mathrm{~cm}^{2}(-17 \%)$ for $37^{\circ}$ and $272 \mathrm{~cm}^{2}(-36 \%)$ for $54^{\circ}$. The value of $\sigma_{\mathrm{XG}}^{\mathrm{TGF}}$ is indicated in Table 1, together with the values for the detectors of RHESSI, Fermi and AGILE, which will be discussed in Sect. 3.

To determine the response of the instrument to electrons (and positrons), we launch 150 mono-energetic beams of $20 \times 10^{6}$ electrons (or positrons). Each beam has a different energy between $20 \mathrm{keV}$ and $20 \mathrm{MeV}$. The electrons are drawn from two sides, at $40^{\circ}$ from nadir and $40^{\circ}$ from zenith, which is representative of an average orientation of a magnetic field line seen by the satellite around equatorial regions (and this is also the orientation of the two IDEE detectors; see next section). The particles are drawn around these two directions with an uniform randomisation of $\pm 30^{\circ}$ for polar an azimuthal angles.

The simulation requires an initial particle to make a deposit of at least $300 \mathrm{keV}$ on a plastic scintillator in order to be detected, or at least $20 \mathrm{keV}$ on a $\mathrm{LaBr}_{3}$ scintillator. These deposits may be due directly to the electrons or from bremsstrahlung, secondary emissions that they are producing.

The effective area of XGRE in comparison to electrons is shown by the black curve of Fig. 2b. There is threshold energy $E_{\mathrm{XG}}^{\mathrm{t}}$ of about $300 \mathrm{keV}$, below which the area is very small (less than $10 \mathrm{~cm}^{2}$ ). Each plastic scintillator is covered by a $0.6 \mathrm{~mm}$-thick hood made of Polyether ether ketone (PEEK). Electrons of $1 \mathrm{MeV}$ kinetic energy will deposit about $E_{\mathrm{XG}}^{\mathrm{t}}$ in these hoods. About $10 \%$ of the area of the hood is covered by a $8 \mathrm{~mm}$-thick stiffener that will absorb more than $\sim 3 \mathrm{MeV}$ of kinetic energy from the electrons when they cross it. For energies higher than $E_{\mathrm{XG}}^{\mathrm{t}}$, the electrons can more likely reach the plastic scintillators because they will scatter to larger distances, and bremsstrahlung emissions (which can be detected by the $\mathrm{LaBr}_{3}$ ) also become more and more important, increasing the effective area from $\approx 100 \mathrm{~cm}^{2}$ at $600 \mathrm{keV}$ to about $720 \mathrm{~cm}^{2}$ at $20 \mathrm{MeV}$. Figure $2 \mathrm{c}$ show the effective area of XGRE against positrons. It is essentially similar to the electron's curve, with the addition of a constant value of about $280 \mathrm{~cm}^{2}$. This constant value is due to positrons that annihilate (into two $511 \mathrm{keV}$ photons) with the detector or some parts of the satellite.

To determine the effective area averaged over a TEB spectrum, $\sigma_{\mathrm{XG}}^{\mathrm{TEB}}$, we apply the method presented in Appendix A. The calculation gives about $233 \mathrm{~cm}^{2}$ for XGRE. This value is presented in Table 1, together with the values obtained for the detectors of RHESSI, Fermi and AGILE, which will be discussed in Sect. 3.

\subsection{The IDEE instrument}

The IDEE instrument is made of two electron detectors from $80 \mathrm{keV}$ to $5 \mathrm{MeV}$ energy. The two main objectives of IDEE are to study Lightning-induced Electron Precipitations (LEP)
Table 1. Summary of the TGF-spectrum averaged ( $\sigma^{\mathrm{TGF}}$ ) and TEBspectrum averaged $\left(\sigma^{\mathrm{TEB}}\right)$ effective areas of RHESSI, AGILE, Fermi and TARANIS.

\begin{tabular}{lcc}
\hline & $\sigma^{\mathrm{TGF}}\left(\mathrm{cm}^{2}\right)$ & $\sigma^{\mathrm{TEB}}\left(\mathrm{cm}^{2}\right)$ \\
\hline RHESSI total & $256^{\mathrm{a}}$ & 74 \\
AGILE-MCAL & $220^{\mathrm{b}}$ & 25 \\
Fermi-GBM BGO (1 unit) & $160^{\mathrm{c}}$ & 21 \\
Fermi-GBM NaI (1 unit) & 33 & 14 \\
Fermi-GBM total & 716 & 350 \\
\hline XGRE & 425 & 233 \\
IDEE & 0 & 22 \\
TARANIS Total & 425 & 255 \\
\hline
\end{tabular}

\footnotetext{
a Ostgaard et al. (2012).

b Marisaldi et al. (2015).

c Briggs et al. (2013).
}

(Voss et al., 1984; Inan et al., 2007) and the electrons beams associated to TGFs, known as TEBs. Two burst-triggering processes run in parallel with these two types of events with different time profiles.

The spectroscopy is possible up to $4.4 \mathrm{MeV}$, and particles depositing more than this energy are counted in an overflow channel. As shown in Fig. 1d, each detector is made of 5 cells of silicon $(\mathrm{Si})$ and 64 cells of cadmium telluride crystal (CdTe). The CdTe cells are mounted in 8 rows by 8 cells format. There are four $\mathrm{Si}$ cells of $1 \mathrm{~cm}^{2}$ each plus one narrow cell of $0.1 \mathrm{~cm}^{2}$ in the centre. The narrow cell is used in regions of high electron fluxes (South Atlantic anomaly, SAA) where others cells will be disabled to significantly decrease the geometrical factor of the sensor. The silicon cells have a total geometrical area of $8 \mathrm{~cm}^{2}\left(4 \mathrm{~cm}^{2}\right.$ per detector $)$ and are $0.3 \mathrm{~mm}$ thick. The CdTe cells are $5 \mathrm{~mm}$ thick and have a total geometrical area of $128 \mathrm{~cm}^{2}$ for the sum of the two IDEE detectors, hence most of the effective area will be due to the CdTe cells.

The two IDEE detection units are pointing $60^{\circ}$ from nadir (IDEE-N) and $60^{\circ}$ from zenith (IDEE-Z) as shown in Fig. $1 b$. The designed viewing angles are $150^{\circ} \times 150^{\circ}$ for electron energies $\gtrsim 600 \mathrm{keV}$ (detected by CdTe cells) and $150^{\circ} \times 40^{\circ}$ for electrons in the range of 80 to $\sim 600 \mathrm{keV}$ (detected by Si cells only). The direction of the detected electrons (hence their pitch angle) can be estimated from coincidence between the Si cells and CdTe cell rows. This will provide important complementary information to the measurements of XGRE.

The effective area vs. energy of IDEE for detecting electrons was estimated with the GEANT4 mass model of the full TARANIS spacecraft using the same methodology as for XGRE. An electron is detected if it deposits at least $80 \mathrm{keV}$ on a $\mathrm{Si}$ cell or at least $350 \mathrm{keV}$ on a CdTe cell. The effective area is presented in Fig. 2b (blue curve). It shows a threshold $E_{\mathrm{I}}^{\mathrm{t}}$ of about $610 \mathrm{keV}$ below which the effective area is essentially due to the Si cells and therefore very small (less than $1 \mathrm{~cm}^{2}$ ). As seen in Fig. 1d, the Si cells are mounted behind 
$\mathrm{a} \sim 4 \mathrm{~mm}$-thick collimator with a narrow slit, allowing only a small fraction of the low-energy electrons to go through the entrance window and reach the Si cells. Notice that two $0.2 \mathrm{~mm}$-thick aluminium fins are also present.

The value of $E_{\mathrm{I}}^{\mathrm{t}}=610 \mathrm{keV}$ is the sum of $350 \mathrm{keV}$ (detection threshold) plus $\approx 260 \mathrm{keV}$, which is the average energy deposited on the $0.65 \mathrm{~mm}$-thick aluminium sheet covering the CdTe cells (see Fig. 1d). The effective area increases to about $28 \mathrm{~cm}^{2}$ at $1.5 \mathrm{MeV}$, when all the electrons can cross the aluminium cover, and then it goes up to about $20 \mathrm{~cm}^{2}$ at $4 \mathrm{MeV}$ and $\approx 38 \mathrm{~cm}^{2}$ at $10 \mathrm{MeV}$, mostly due to the scattering of the particles over a larger distance, and remains constant for higher energies.

The effective area averaged over a TEB spectrum, $\sigma_{\mathrm{I}}^{\text {TEB }}$, can be calculated to be about $22 \mathrm{~cm}^{2}$ (see Appendix A for the method used). If IDEE or XGRE trigger a TGF or a TEB event, they will be able to trigger the other instrument: we should then consider the TARANIS spacecraft a detector of TEB with an averaged effective area $\sigma_{\mathrm{T}}^{\mathrm{TEB}}$ of about $255 \mathrm{~cm}^{2}$.

\section{Comparison between instruments}

In this section we present a comparison of the performances of XGRE and IDEE with those of RHESSI, Fermi-GBM and AGILE-MCAL in the context of TGFs, without including CGRO-BATSE. CGRO-BATSE detected 79 TGFs between the years 1991 and 2000, some of which are clearly identified as TEBs (Dwyer et al., 2008). However, we did not include them because this number of TGFs is significantly smaller than for RHESSI, Fermi-GBM and AGILE. Furthermore, BATSE was only triggered on long events (it had a trigger window that could not be lower than $64 \mathrm{~ms}$ ), overestimated duration and underestimated brightness (Grefenstette et al., 2008; Gjesteland et al., 2010), thus making it much harder to separate TGF and TEB events compared to the other instruments.

\subsection{TGF detection performance}

The Reuven Ramaty High Energy Solar Spectroscopic Imager (RHESSI) is a NASA spacecraft designed for the study of high-energy radiation from the sun. It uses an array of nine high-purity germanium detectors cooled down to liquid nitrogen temperature. A detailed description of the detector is presented in Lin et al. (2002); Smith et al. (2002). A response matrix of the RHESSI detector in the TGF context is publicly available (see Sect. 7 on data availability). The provided matrix is already averaged for the spacecraft position and attitude. From this matrix, we can deduce the effective area vs. energy of the detector, which is presented in Fig. 2a. As indicated in Ostgaard et al. (2012), RHESSI has an effective area for detecting TGF $\sigma_{\mathrm{R}}^{\mathrm{TGF}}$ of about $256 \mathrm{~cm}^{2}$.

The Astro-Rivelatore Gamma a Immagini Leggero (AGILE) is a satellite from the Italian Space Agency dedicated to the study of high-energy gamma rays (typically above $50 \mathrm{MeV}$ ) in the universe. The mini-calorimeter (MCAL) detector uses 30 cesium iodide (CsI) scintillator bars and can be used to detect lower-energy gamma rays (above $400 \mathrm{keV}$ ). It is presented in detail in Tavani et al. (2009); Labanti et al. (2009). The effective area vs. energy for AGILE-MCAL is taken from Marisaldi et al. (2015) and reproduced in Fig. 2a. As indicated in the same article, AGILE-MCAL has an effective area for detecting TGF $\sigma_{\mathrm{A}}^{\mathrm{TGF}}$ of $220 \mathrm{~cm}^{2}$.

The Gamma-Ray Burst Monitor (GBM) on board the Fermi spacecraft is presented into details in Meegan et al. (2009). GBM is made of 12 sodium iodide (NaI) cylindrical detectors which are sensitive in the $20 \mathrm{keV}-10 \mathrm{MeV}$ energy range, and two bismuth germinate (BGO) cylindrical detectors that are sensitive from $200 \mathrm{keV}$ to $40 \mathrm{MeV}$. Regarding the $\mathrm{NaI}$ detectors, the photons above $1 \mathrm{MeV}$ are counted in a single channel and not used for spectroscopy, but they are included for the TGF counts and the search algorithm. The effective area for high-energy photons can be calculated from an average of response matrices generated by the gbmrspgen tool, developed by the Fermi-GBM collaboration (see Sect. Data availability) The response matrix of GBM for a given event depends on the position and attitude of the spacecraft. To get an average effective area of the GBM detectors, we calculated an average matrix from 94 matrices that were generated from the 94 GBM-triggered TGF of 2013. The effective area vs. energy of the BGO and NaI detectors are presented in Fig. 2a.

As presented in Briggs et al. (2013), the effective area that should be used to detect TGF is $160 \mathrm{~cm}^{2}$ for each BGO detector. Our calculation from the response matrices show that it should be about $33 \mathrm{~cm}^{2}$ for each NaI detector, giving a total $\sigma_{\mathrm{F}}^{\mathrm{TGF}}$ of about $716 \mathrm{~cm}^{2}$ for Fermi-GBM. A summary of these averaged effective areas for detecting TGF is presented in Table 1.

Below $30 \mathrm{keV}$, the NaI detectors of Fermi-GBM have the best effective area that ranges between 40 and $300 \mathrm{~cm}^{2}$. However, from simulation results it is not expected that TGF detected at satellite altitude shows many photons at these energies (Sarria et al., 2015), though this part of the spectrum has not yet been properly detected. Between 30 and $220 \mathrm{keV}$, XGRE has the best effective area $\left(350-850 \mathrm{~cm}^{2}\right)$, which is about 1.4 higher than Fermi-NaI detectors and five times higher than RHESSI. For higher energies, it falls below AGILE-MCAL and Fermi-BGOs at about $1 \mathrm{MeV}(\sim$ $\left.280 \mathrm{~cm}^{2}\right)$ and below RHESSI at about $2 \mathrm{MeV}\left(\sim 250 \mathrm{~cm}^{2}\right)$. From around $760 \mathrm{keV}$, the effective area of AGILE-MCAL increases greatly and reaches about $750 \mathrm{~cm}^{2}$ at $20 \mathrm{MeV}$, making it about twice as good than RHESSI and FermiBGOs and three times better than XGRE. The uncertainties on the effective area values presented in this section are discussed in Appendix B.

RHESSI, Fermi and AGILE suffered from issues related to the fact that their design is not perfectly suited to detect very bright and short events such as TGFs. Concerning AG- 
ILE, this issue was likely solved after the deactivation of its anti-coincidence shield (Marisaldi et al., 2015). Depending of the processing algorithms and the electronics used by the detector, this can cause several issues, such as underestimating the number of counts for bright TGFs (because of the detector's "dead time"), overestimating the duration of bright TGFs, or incorrectly measuring photon energies (because of pulse "pile-up").

For Fermi GBM detectors, the nominal dead time lasts $2.6 \mu \mathrm{s}$, but it goes up to $10.4 \mu \mathrm{s}$ if the overflow channel is filled; i.e. there is a count with energy above $1 \mathrm{MeV}$ on a NaI detector or a count with energy $>40 \mathrm{MeV}$ on a BGO detector. NaI detectors have a high rate of overflow counts, making TGF spectra obtained from them very hard to analyse in practice. On the other hand, these problems are less important for the BGO detectors, allowing correction and study of spectra from single TGF events (Mailyan et al., 2016).

XGRE uses lanthanum bromide crystal scintillators coupled with fast electronics, resulting in a dead time of $350 \mathrm{~ns}$ and a pile-up time of $150 \mathrm{~ns}$, making it capable of counting up to $\sim 9$ photons $\mu \mathrm{s}^{-1}$ (each of the three sensors being independent), which should be enough to avoid dead time or pile-up issues up to a count rate of about 10 million counts per second. Thus XGRE should derive precise measurements of light curves and spectra, even for the shortest TGF.

The dead time of IDEE is less than $4 \mu$ s. It should not suffer from significant dead times issues when detecting TEBs, since they show about 20 times fewer particles $\mathrm{cm}^{-2} \mathrm{~ms}^{-1}$ at satellite altitude compared to TGFs (see Fig. 3), and IDEE also has a relatively small effective area.

The TGF detection methods used by RHESSI, FermiGBM and AGILE-MCAL are described respectively in Gjesteland et al. (2012); Briggs et al. (2013); Marisaldi et al. (2014). Concerning XGRE, a TGF event can be triggered if a given number of counts is reached within a $10 \mu$ s window. This number can be changed between half-orbits and will be comprehensively tested during the spacecraft commissioning phase. There is also another trigger window working the same way but with a duration of $100 \mu$ s. It is made for the detection of (short) gamma-ray bursts, but it is not excluded it could be triggered on some TGF or TEB events. Particles detected prior to the trigger timer are saved (with an adjustable number). All the particles detected after the trigger time are saved until the TGF ends.

The IDEE instrument uses a dynamical algorithm which evaluates a floating background (in preselected energy range and $\mathrm{Si} / \mathrm{CdTe}$ cell type) over selected time periods, based on Poisson distribution assumption and using a dynamic threshold. The IDEE instrument will be triggered on TEB events, which will also trigger all the instruments of the TARANIS payload (including XGRE).

\subsection{TEB detection performance}

RHESSI, Fermi-GBM and AGILE-MCAL were not designed to detect electrons or positrons, therefore no response matrix is provided for these particles. Nevertheless, we could proceed to Monte Carlo simulations of these detectors to get a basic idea of their performances for detecting TEBs.

The RHESSI detectors are surrounded by several millimetres of aluminum (Dwyer et al., 2012), which only very highenergy electrons can cross. Using a complete mass model of the RHESSI spacecraft (D. Smith, personal communication, 2016), we could estimate its effective area for different electron incident energies. The procedure we followed is different to that from XGRE/IDEE, since the orientation (attitude) of the spacecraft is not known and it has no reason to point towards Earth like TARANIS. Therefore we simply draw the particles randomly and uniformly over all directions around the spacecraft.

The effective area of RHESSI against electrons is displayed in Fig. 2b. It is $<1 \mathrm{~cm}^{2}$ below $400 \mathrm{keV}$, it rises to about $4 \mathrm{~cm}^{2}$ at $1 \mathrm{MeV}$ and then increases with energy until it reaches about $500 \mathrm{~cm}^{2}$ at $20 \mathrm{MeV}$ due to significant bremsstrahlung emissions. Using the same method as for XGRE, we could estimate an effective area $\sigma_{\mathrm{R}}^{\mathrm{TEB}}$ of RHESSI averaged for a typical TEB event of about $74 \mathrm{~cm}^{2}$.

Regarding Fermi-GBM, GEANT4 detailed models of single BGO and NaI detectors are available as GDML files as part of the GRESS software (Kippen et al., 2007). A NaI detector is covered by aluminium parts (including the photomultiplier tube), and one side of the crystal has a $0.2 \mathrm{~mm}$ thick beryllium window and a $0.7 \mathrm{~mm}$-thick silicone layer in between them. The BGO detector has some dense parts on both sides (including the photo-multiplier tubes) and the rest is covered with a $\sim 3 \mathrm{~mm}$-thick carbon fibre (CRFP) and maintained by two titanium rings.

These single detector models are not enough to estimate the response of Fermi-GBM to electrons, because they do not take into account their accommodation on the spacecraft, nor the entire spacecraft (e.g. platform, subsystems and LAT detector). We could not have access to the full mass model of the Fermi satellite, but we could build a very simplified version, by looking to several Fermi-GBM documents; in particular Meegan et al. (2009) and the references therein. Our simplified model contains the biggest parts of the spacecraft with approximative densities, and the $2 \mathrm{BGO}$ and $12 \mathrm{NaI}$ detectors are accurately placed. We think this model is reasonable for electrons since they get easily absorbed by the elements of the spacecraft, and also we only need a basic estimation of the GBM response to electrons.

The response of GBM to mono-energetic electron beams is presented in Fig. 2b. We followed the same procedure as for RHESSI (the particles are drawn randomly and uniformly over all directions around the spacecraft). The effective areas show threshold energies $\left(E_{\mathrm{NaI}}^{\mathrm{t}} \approx 500 \mathrm{keV}, E_{\mathrm{BGO}}^{\mathrm{t}} \approx\right.$ $1.5 \mathrm{MeV}$ ) below which the effective area is very small. Below 
these energies, the electrons or positrons can hardly reach the crystals, because they are absorbed by surrounding materials. Above these threshold energies, the leptons have enough energy to have a chance of reaching the crystals, and the effective area increases with increasing kinetic energy. This increase is because electrons with higher energy will scatter to higher distances in the spacecraft and will also produce more bremsstrahlung photons with higher energies. For $20 \mathrm{MeV}$ electrons, it reaches a value of about $770 \mathrm{~cm}^{2}$ for the sum of the $12 \mathrm{NaI}$ and $325 \mathrm{~cm}^{2}$ for the sum of the two BGO. The response to the positron is similar to the response to electrons, with the addition of a constant value, which is about $690 \mathrm{~cm}^{2}$ in this case. As for other instruments, we can use the method presented in Appendix A to calculate a TEB-averaged effective area for Fermi-GBM, which is $\sigma_{\mathrm{F}}^{\mathrm{TEB}} \approx 350 \mathrm{~cm}^{2}$

Regarding AGILE, the full mass model was provided by the AGILE team (M. Marisaldi, personal communication, 2016). The MCAL detector on the AGILE spacecraft is surrounded by several elements (e.g. the MITA spacecraft Bus, the GRID, the Super-AGILE, the anticoincidence system or the carbon fibre structure surrounding the CsI bars) that will absorb a significant amount of energy of the electrons before they can reach the CsI crystals (Longo et al., 2002; Cocco et al., 2002; Labanti et al., 2009). We were able to perform a simulation to check the response of MCAL to electron and positron beams, following the same procedure as for RHESSI and Fermi-GBM. The results are displayed in Fig. $2 \mathrm{~b}$ and c. (red curves). All the incident electrons with kinetic energies below about $3 \mathrm{MeV}$ are absorbed before reaching the CsI bars. Above this energy, the effective area increases with increasing energy, mainly due to the production of bremsstrahlung photons that can reach the detectors. It reaches $\sim 430 \mathrm{~cm}^{2}$ at $20 \mathrm{MeV}$, where many bremsstrahlung photons are produced. As for the other instruments, the response to positron is similar to the response to electrons, with the addition of a constant value (about $100 \mathrm{~cm}^{2}$ in this case) due to photons produced by positron annihilation with the spacecraft. It results in an effective area $\sigma_{\mathrm{A}}^{\mathrm{TEB}}$ averaged on a TEB spectrum of about $25 \mathrm{~cm}^{2}$ for AGILE-MCAL. All the TGF and TEB effective areas are summarised in Table 1. The uncertainties on the effective area values presented in this section are discussed in Appendix B.

For all these detectors, dead times and pile-up effects are not a big issue concerning TEB detection. Indeed, the flux (particles $\mathrm{cm}^{-2} \mathrm{~ms}^{-1}$ ) for a TEB event at satellite altitude is usually about 20 times less than for TGF (see Fig. 3), and their averaged effective area and are also several times smaller for electrons than for gamma rays.

\section{Estimating TGF/TEB detection rates}

\subsection{Past TGF and TEB detections}

The AGILE TGFs of the second catalogue are given between 23 March 2015 and 23 June 2015, and contains 279 TGFs (Marisaldi et al., 2015). Taking into account that TGFs are slightly more likely to be detected during this time period than the average of the rest of the year, it corresponds to about $N_{\mathrm{A}}=1070 \mathrm{TGFs}_{\mathrm{yr}}{ }^{-1}$. For RHESSI, the detection rate is about $N_{\mathrm{R}}=350 \mathrm{TGF} \mathrm{yr}^{-1}$ for the second catalogue (Ostgaard et al., 2015). For Fermi, by looking to publicly available catalogue (http://fermi.gsfc.nasa. gov/ssc/data/access/gbm/tgf/), we could estimate that about $N_{\mathrm{F}}=650 \mathrm{TGFs} \mathrm{yr}^{-1}$ were detected after the offline searching method was set up (Briggs et al., 2013). All these values are summarised in Table 2.

Concerning terrestrial electrons beams (TEBs), they were detected by RHESSI and Fermi. RHESSI clearly detected only two TEB events and one of them was presented (Smith et al., 2006). This number is too low to permit an estimation of the number of TEB event that will be detected by TARANIS. As discussed in the previous section, Fermi-GBM has a much better sensitivity to electrons than RHESSI and detected about 24 events between August 2008 and February 2015, giving $3.7 \mathrm{TEBs}^{-1} \mathrm{r}^{-1}$.

No TEB event was reported by AGILE, and we speculate that this is because the effective area for detecting TEB is not high enough $\left(\approx 25 \mathrm{~cm}^{2}\right)$ and is actually mostly due to bremsstrahlung or annihilation photons produced by the electrons/positrons (see previous section).

\subsection{Simulated flux profiles}

Using the MC-PEPTITA Monte Carlo model (Sarria et al., 2015), we estimated average flux profiles of gamma rays and electrons detected by the satellites and associated to TGFs. The source is assumed to follow an energy spectrum $\propto 1 / E \times \exp (-E /(7.3 \mathrm{MeV}))$. The production altitude is uniformly sampled between 12 and $15 \mathrm{~km}$ and it is located at $\left(\theta=-13^{\circ}, \phi=32^{\circ}\right)$ geodetic coordinates. The opening angle is uniformly sampled between 0 and $40^{\circ}$. The source is also tilted by an angle $\psi$ that is uniformly sampled between 0 and $10^{\circ}$.

Concerning the time distribution of the source, there are currently two different results. On one hand, by comparing simulated TGFs with AGILE data, Marisaldi et al. (2015) suggests that, at the source, the TGF is created almost instantaneously, so that the TGF durations are mainly due to delays due to scatterings in the atmosphere, and long-duration TGFs may be a succession of multiple pulses. On the other hand, by comparing simulated TGFs with Fermi data, Fitzpatrick et al. (2014) concluded that the source distribution is not created instantaneously for a vast majority of FermiGBM TGFs and indicated that a good fit to the Fermi data 
is a time distribution of the TGF source following a Gaussian (normal) distribution with $\sigma=50 \mu$ s. The results of MCPEPTITA simulations suggest a source duration in between an almost instantaneous source and a normal source duration of $\sigma=50 \mu \mathrm{s}$. Assuming a normal distribution definition $\propto \exp \left(-t^{2} /\left(2 \sigma^{2}\right)\right)$ for the TGF photons when they are produced and using a SD of $\sigma=20 \mu$ s results in $t_{90}$ durations of TGFs down to $\sim 60-70 \mu$ s; this corresponds to the lowest durations observed by the Fermi spacecraft (Fitzpatrick et al., 2014).

From Fermi data, Fitzpatrick et al. (2014) indicate that Fermi typically detects about 0.08 photons $\mathrm{cm}^{-2}$ over a TGF duration of $200 \mu \mathrm{s}$ (giving 0.4 photons $\mathrm{cm}^{-2} \mathrm{~ms}^{-1}$ ) at a radial distance of $500 \mathrm{~km}$ between the satellite position and the position of the source of the TGF. We used this value to give a scale to the flux distributions presented in Fig. 3, which is obtained by assuming that $4.4 \times 10^{17}$ photons (with energies $>20 \mathrm{keV}$ ) are produced at the source.

The flux profiles resulting from the simulations are presented in Fig. 3a. The fluxes are presented as a function of the radial distance $\left(r_{\mathrm{d}}\right)$ between the source position of the TGF (projected at the altitude of the satellite) and the satellite. The three presented altitudes approximately correspond to AGILE $(490 \mathrm{~km})$, Fermi $(550 \mathrm{~km})$ and TARANIS $(700 \mathrm{~km})$. The fluxes are expressed in terms of particles $\mathrm{cm}^{-2} \mathrm{~ms}^{-1}$, considering photons and electrons. Figure $3 \mathrm{~b}$ presents the corresponding time durations $\left(t_{90}\right)$.

Below $r_{\mathrm{d}}=100 \mathrm{~km}$, the photon flux at an altitude of $490 \mathrm{~km}$ is about $31 \%$ higher than the flux at $560 \mathrm{~km}$, and twice the flux at $700 \mathrm{~km}$. This difference of fluxes corresponds to the $1 / R^{2}$ variation expected from an isotropic point source detected from various distances. At about $r_{\mathrm{d}}=$ $300 \mathrm{~km}$, the photon fluxes are similar for the three altitudes. Above $r_{\mathrm{d}} \approx 600 \mathrm{~km}$, the flux at $700 \mathrm{~km}$ is about $57 \%$ higher than the flux at $560 \mathrm{~km}$, and the flux at $560 \mathrm{~km}$ is $39 \%$ times higher than at $490 \mathrm{~km}$ altitude.

Concerning electrons, the fluxes are close at the three considered altitudes, so we only represented the flux at $550 \mathrm{~km}$. It is important to note that the time scattering of electrons detected at satellite altitude can vary significantly depending on the length of the magnetic field line the particles have to travel, which depends on the coordinates of the spacecraft (higher absolute latitudes usually meaning longer field lines). This time dispersion is because electrons are produced with various pitch angles and energies, which will imply various propagation speeds along the geomagnetic field lines (Dwyer et al., 2008; Sarria et al., 2016). The results shown here are for a given magnetic field line of about $6000 \mathrm{~km}$ length, which can be roughly considered an average TEB case. In Fig. 3, the flux of electrons is about 3 times higher if detected in the hemisphere where the TGF is produced. The spatial fluxes (electrons $\mathrm{cm}^{-2}$ ) are actually quite close in both hemispheres, but, as showed in Fig. $3 b$, the $t_{90}$ time durations of the TEBs are about 3 times higher in the opposite hemisphere.

\subsection{Estimating a map of TGFs that can be detected by satellites}

An approximative map of TGFs that can be detected by satellites was built, based on the TRMM-LISS and OTD global lightning density map (Cecil et al., 2014). Compared to this distribution, it was noticed that the TGF density detected by satellites tends to be higher towards the equator. This is supposed to be due to the fact that the tropopause is higher for latitudes closer to zero, where TGF photons have to cross less atmosphere before reaching space, and can be more easily detected by satellites. Let $\rho_{\mathrm{L}}(\theta, \phi)$ be the lightning density from the LISS/OTD database for a given latitude and longitude. Let $T(\theta)$ be an approximative profile of the tropopause height as function of latitude. We used data obtained from Lewis (2009), which were fit by a simple normal distribution $T(\theta) \propto \exp \left[\left(\left(\theta-\theta_{0}\right) / 2 \sigma_{\theta}\right)^{2}\right]$ with parameters $\theta_{0}=0.8746^{\circ}$ and $\sigma_{\theta}=37.49^{\circ}$. Then the TGF density at a given latitude and longitude is assumed as follows:

$\rho_{\mathrm{TGF}}(\theta, \phi) \propto \rho_{\mathrm{L}}(\theta, \phi) \times[T(\theta)]^{\beta}$.

The proportionality sign $(\propto)$ denotes the fact that we do not define an absolute scale for this density, and therefore all the estimations given afterwards will only use ratios of summed values of $\rho_{\mathrm{TGF}}(\theta, \phi)$. Equation (1) shows that the tropopause profile is set at a power $\beta$ that we are using as a free parameter, and will be adjusted to get the best possible agreement between this simple model and the observations. This estimated TGF global production map is displayed in Fig. 4. This figure also shows the ground tracks of RHESSI, Fermi, AGILE and TARANIS (planned). They have been calculated using the two-line element from the CELESTRACK database (https://www.celestrak.com/NORAD/ elements/). The orbit of TARANIS is assumed to be similar to that of the DEMETER satellite. RHESSI, Fermi and AGILE show equatorial orbits with various inclinations (38, 25.6 and $2.5^{\circ}$ respectively), whereas TARANIS will follow a quasi-polar sun-synchronous orbit. This orbit is not the best for detecting TGFs since it will have a significantly reduced coverage of the equatorial region where TGF are more likely to be detected, and the impacts on TGF detection rates are discussed in the next section. However, the orbit of TARANIS covers all latitudes almost uniformly and should permit a global distribution of TGF to be determined without orbital bias.

\subsection{Estimating TGF and TEB detection rates}

Each detector has a minimal threshold of counts $n_{\mathrm{X}}^{\min }$ so that any detected event must be significantly above the background level to be considered as a TGF. Gjesteland et al. (2012) indicate that $n_{\mathrm{R}}^{\min }=11$ (for the RHESSI second catalogue) and Ostgaard et al. (2012) indicate $n_{\mathrm{F}}^{\min }=19$ for Fermi-GBM. The value for AGILE $\left(n_{\mathrm{A}}^{\min }=10\right)$ is found by the TGF to have the lowest number of count in the second 
(a)

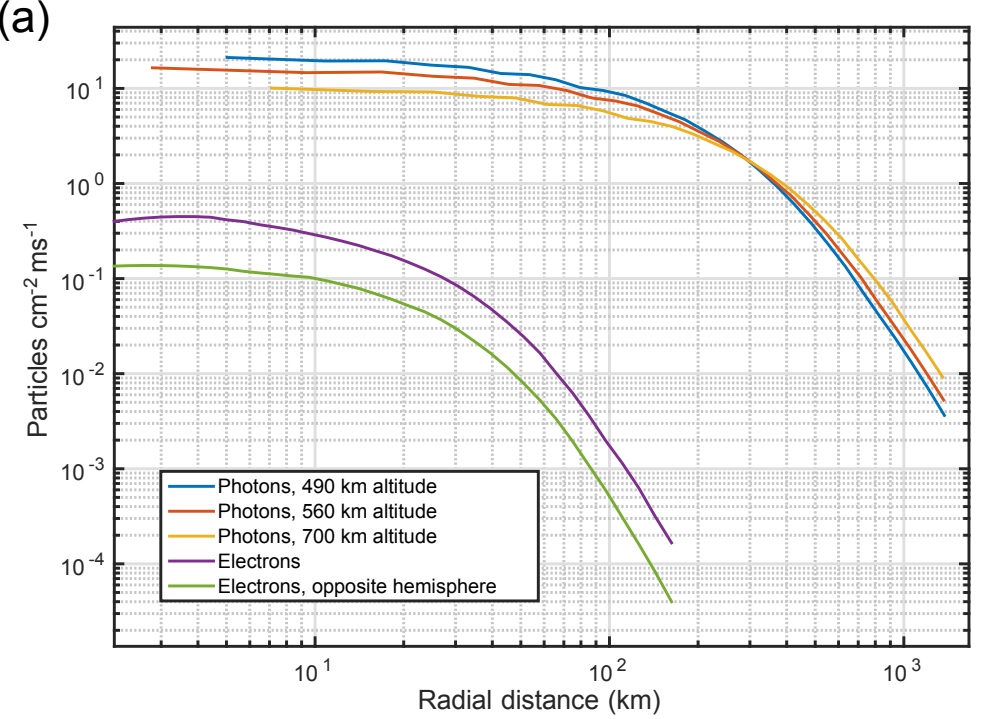

(b)

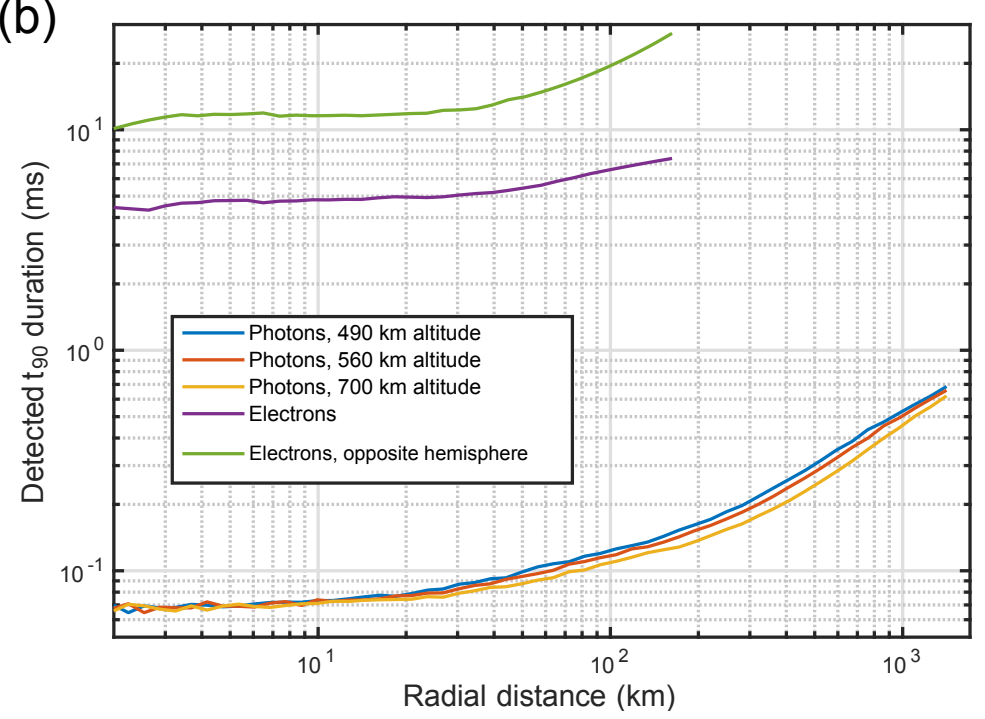

Figure 3. Results of MC-PEPTITA simulations. (a) Flux profile vs. radial distance between the TGF source (projection at satellite altitude) and the satellite for photons (various altitudes) and electrons (seen at the hemisphere of production and in the opposed hemisphere). The two electron profiles correspond to a detection altitude of $560 \mathrm{~km}$, but are very close for 490 or $700 \mathrm{~km}$ altitude. (b) $t_{90}$ durations associated to the flux profiles. Like for the flux profiles, the electron distributions stay very similar at 490 or $700 \mathrm{~km}$ altitude.

Table 2. Altitudes, detection count thresholds, limit radii, detection efficiencies and number of TGFs per year (observed and estimated) for the considered satellites.

\begin{tabular}{lcccccc}
\hline & $\begin{array}{c}h_{\mathrm{X}} \\
(\mathrm{km})\end{array}$ & $\begin{array}{c}n_{\mathrm{X}}^{\min } \\
(\text { counts })\end{array}$ & $\begin{array}{c}R_{\mathrm{X}}^{\mathrm{lim}} \\
(\mathrm{km})\end{array}$ & $E_{\mathrm{X} / \mathrm{A}}$ & $\begin{array}{c}N_{\mathrm{X}}^{\mathrm{TGF}, \mathrm{obs}} \\
\left(\mathrm{TGFs} \mathrm{yr}^{-1}\right)\end{array}$ & $\begin{array}{c}N_{\mathrm{X}}^{\mathrm{TGF}, \mathrm{est}} \\
\left(\mathrm{TGFs} \mathrm{yr}^{-1}\right)\end{array}$ \\
\hline $\begin{array}{l}\text { RHESSI } \\
\begin{array}{l}\text { (Second catalogue }) \\
\text { Fermi-GBM }\end{array}\end{array}$ & 565 & 11 & 694 & $32.7 \%$ & 350 & 349.7 \\
$\begin{array}{l}\text { AGILE-MCAL } \\
\text { (Second catalogue })\end{array}$ & 491 & 10 & 795 & $60.7 \%$ & 650 & 649.2 \\
TARANIS-XGRE & 700 & $10 ?$ & 820 & $24.9 \%$ & $?$ & 1070 \\
\hline
\end{tabular}

a Takes into account the diurnal correction. 


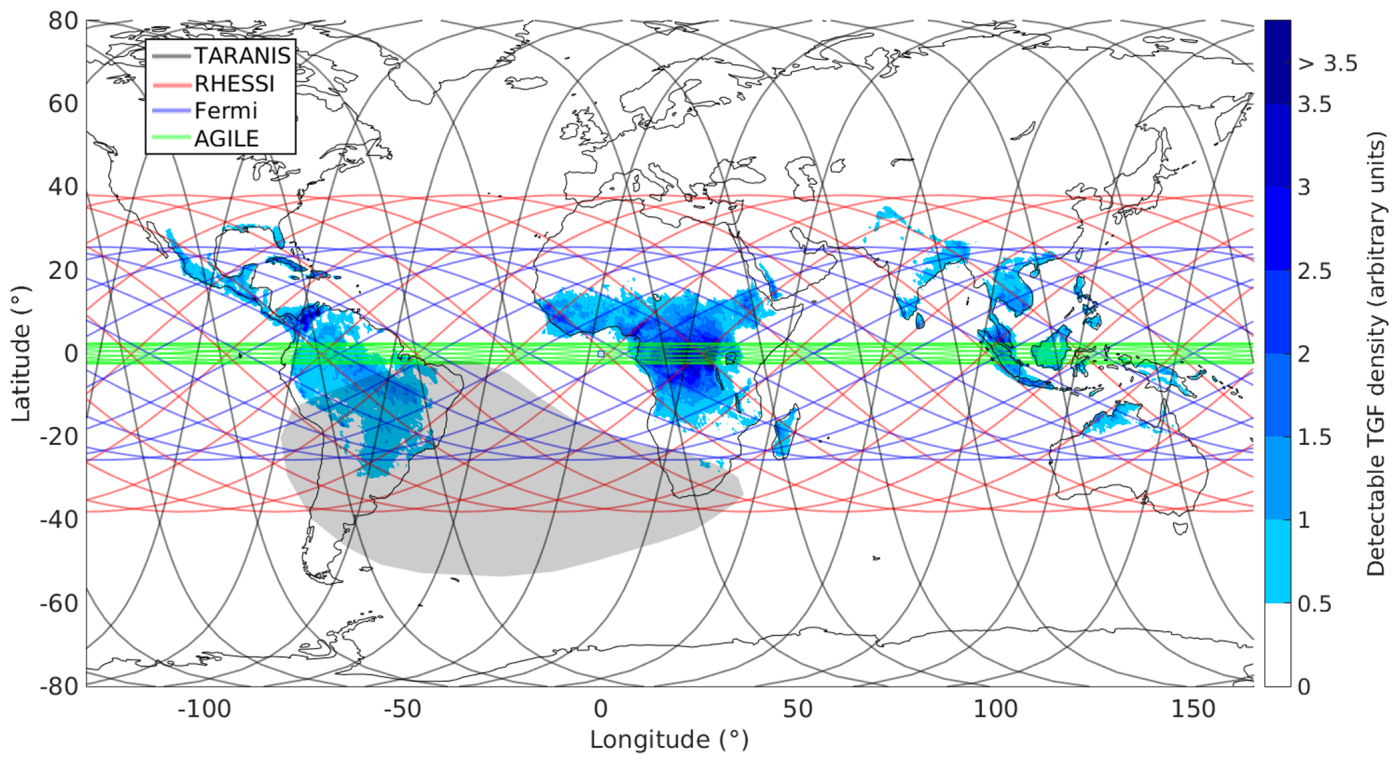

Figure 4. Estimated global detectable TGF density map, and ground tracks of the orbits of TARANIS (planned), RHESSI, Fermi and AGILE. The grey area denotes an approximative South Atlantic anomaly assumed for the simulations, and where TGF can occur but no detection by satellites is possible due to high background.

Table 3. Count thresholds, limit radii, detection efficiencies and number of TEBs per year (observed and estimated) for the considered satellites.

\begin{tabular}{lccccc}
\hline & $\begin{array}{c}n_{\mathrm{X}}^{\min } \\
(\text { counts })\end{array}$ & $\begin{array}{c}R_{\mathrm{X}}^{\lim } \\
(\mathrm{km})\end{array}$ & $E_{\mathrm{X} / \mathrm{F}}$ & $\begin{array}{c}N_{\mathrm{X}}^{\mathrm{TEB}, \text { obs }} \\
\left(\mathrm{TEBs} \mathrm{yr}^{-1}\right)\end{array}$ & $\begin{array}{c}N_{\mathrm{X}}^{\mathrm{TEB}, \mathrm{est}} \\
\left(\mathrm{TEBs} \mathrm{yr}^{-1}\right)\end{array}$ \\
\hline Fermi-GBM & $\sim 150$ & 23 & $100 \%$ & 3.7 & 3.7 \\
TARANIS & $10 ?$ & 72 & $885 \%$ & $?$ & $25^{\mathrm{a}}$ \\
$(\mathrm{XGRE}+$ IDEE $)$ & & & & & \\
\hline
\end{tabular}

a Takes into account the diurnal correction.

AGILE catalogue (Marisaldi et al., 2015). The value of $n_{\mathrm{G}}^{\min }$ for TARANIS-XGRE is also assumed to be 10 but is hard to predict and will depend on the in-flight background. We should wait for the instrument to be launched so we can know precisely which value will be used. Appendix B discusses how variations on $n_{\mathrm{T}}^{\min }$ affect the results presented in this section.

The ratio between this threshold value $n_{\mathrm{X}}^{\min }$ and the averaged effective area $\sigma_{\mathrm{X}}^{\mathrm{TGF}}$ gives a limit of sensitivity for the instrument. Combining this limit with the radial distance flux profiles (Sect. 4.2 and Fig. 3), we can deduce a limit distance $R_{\mathrm{X}}^{\lim }$ corresponding to this sensitivity. The limit of sensitivity of Fermi is $\approx 0.053$ photons $\mathrm{cm}^{-2} \mathrm{~ms}^{-1}$ ( $=19$ photons $\left./ 716 \mathrm{~cm}^{2} / 500 \mu \mathrm{s}\right)$ and it corresponds to a limit radius of $R_{\mathrm{F}}^{\lim } \approx 795 \mathrm{~km}$. This value is consistent with the maximum distance between the TGF source positions and Fermi footprints given using WWLLN (World Wide Lightning Location Network) associations (Briggs et al., 2013; Fitzpatrick et al., 2014). We can also determine $R_{\mathrm{R}}^{\text {lim }}=$ $694 \mathrm{~km}$, which is reasonably close to the largest distance found between RHESSI's position and the WWLLN match of the TGF source location (Nisi et al., 2014). Using the simulated photon flux and time profiles at $490 \mathrm{~km}$ altitude, we can estimate $R_{\mathrm{XG}}^{\lim }=648 \mathrm{~km}$ for AGILE-MCAL. Using the simulated photon flux and time profiles at $700 \mathrm{~km}$ altitude, we could estimate $R_{\mathrm{XG}}^{\lim }=820 \mathrm{~km}$ for TARANISXGRE (corresponding to a t90 duration of about $420 \mu \mathrm{s}$ ). All theses values are summarised in Table 2.

Knowing $R_{\mathrm{X}}^{\lim }$, the orbit of the satellite, and the detectable TGF density map, we can deduce a detection efficiency, expressed as $E_{\mathrm{X} / \mathrm{A}}$. Since we did not find an absolute scale for it, we only expressed it relatively to AGILE (second catalogue), which has the highest TGF detection rate.

This detection efficiency is computed using the following algorithm:

- We consider a step of time $\delta_{t}=120 \mathrm{~s}$, which is small enough compared to the scale of duration of one orbit of about $5400 \mathrm{~s}$. 
- Each time step corresponds to a position of the satellite $\left(\theta_{\mathrm{i}}^{\mathrm{X}}, \phi_{\mathrm{i}}^{\mathrm{X}}\right)$.

- At each position, the TGF densities from the map $\left(\rho_{\mathrm{TGF}}(\theta, \phi)\right)$ are summed within a radius of $R_{\mathrm{lim}}^{\mathrm{X}}$ around $\left(\theta_{\mathrm{i}}^{\mathrm{X}}, \phi_{\mathrm{i}}^{\mathrm{X}}\right)$, giving a quantity $\Sigma^{\mathrm{X}}$.

$-\Sigma^{\mathrm{X}}$ is incremented this way over $48 \mathrm{~h}$ (1442 steps).

- If $\left(\theta_{\mathrm{i}}, \phi_{\mathrm{i}}\right)$ is inside the South Atlantic anomaly (SAA), $\Sigma^{\mathrm{X}}$ is not incremented. We use the approximative SAA area presented as a grey area in Fig. 4.

The ratio $\Sigma^{\mathrm{X}} / \Sigma^{\mathrm{A}}$ (between a given satellite $\mathrm{X}$ and $\mathrm{AG}$ ILE) gives a detection efficiency $E_{\mathrm{X} / \mathrm{A}}$, the values of which are summarised in Table 2. Applying these efficiencies to the AGILE detection rate gives detection rate estimates of $649.2 \mathrm{TGFs} \mathrm{yr}^{-1}$ for Fermi and $349.7 \mathrm{TGFs} \mathrm{yr}^{-1}$ for RHESSI. For both, the relative differences with the observed detection rates are less than $1 \%$. Note that the value of the parameter $\beta$ for the detectable TGF map (see Sect. 4.3) was adjusted to $\beta=7.1$ in order to minimise these differences.

One last parameter to be taken into account is the diurnal cycle of lightning. Lightning activity was found to be non-uniform with local time and is maximum around $17 \mathrm{~h}$ and minimum around $11 \mathrm{~h}$ (Cecil et al., 2014). TARANIS, with its sun-synchronous orbit will always be at a local time between 22:30-02:00 and 10:30-14:00 LT. The other satellites have equatorial orbits; therefore their local time is almost uniformly distributed between 00:00 and 24:00 LT. It implies that XGRE will miss an extra $24 \%$ of TGF compared to the other satellites. Finally, our estimation gives about 200 TGFs $\mathrm{yr}^{-1}$ for TARANIS. All the important parameters used for this estimation are summarised in Table 2.

The catalogue of Fermi GBM TEBs presents 24 events between 8 July 2008 and 2 February 2015, giving $N_{\mathrm{F}}=$ 3.7 TEBs $\mathrm{yr}^{-1}$. These events present a minimum count of $n_{\mathrm{F}}^{\min } \approx 150$ for the event ID TEB130521580. Since XGRE will discriminate electrons from photons, this threshold should be similar to TGF, i.e. $n_{\mathrm{XGRE}}^{\min }=10$. As in the case of TGFs, this value is hard to estimate and the correct value will only be known after in-flight tests of the instrument. As discussed in Sect. 4.2, the effective area of TARANIS (XGRE + IDEE) for detecting TEB could be estimated: $\sigma_{\mathrm{G}}^{\mathrm{TEB}}=255 \mathrm{~cm}^{2}$. This TEB average effective area could be estimated for Fermi-GBM, giving $\sigma_{\mathrm{R}}^{\mathrm{TEB}} \approx 350 \mathrm{~cm}^{2}$.

To determine the TEB detection efficiency, the algorithm presented for the TGF case has to be modified. If the satellite is located at given coordinates, the considered density is not the density at this point, but the sum of the two densities located at the two magnetic footprints of the field line. These coordinates are determined from MC-PEPTITA runs that can track the electrons in the geomagnetic field. In these simulations, the electrons are drawn at $100 \mathrm{~km}$ altitude with various pitch angles (this altitude being approximately the altitude where the secondary electrons from TGF can escape the Earth's atmosphere).
From all this information, we can calculate detection efficiencies between TARANIS and Fermi $E_{\mathrm{T} / \mathrm{F}}^{\mathrm{TEB}}=885 \%$. As for the TGF estimation, we also have to account for the diurnal correction for the TARANIS case, and our final estimate is about $25 \mathrm{TEBs} \mathrm{yr}^{-1}$. All the important parameters used for this estimation are summarised in Table 3.

\section{Conclusions}

The TARANIS spacecraft will have two important instruments with which to study TGFs and TEBs: XGRE and IDEE. XGRE will detect both electrons and X-rays and gamma rays, with the ability to discriminate one type of particle from the other. The IDEE instrument is focused on electrons, with the ability to estimate their pitch angle. The instruments will be able to trigger one another.

Using Monte Carlo simulations, mass models and a standard TGF spectrum, we can estimate that XGRE will have an effective area of about $425 \mathrm{~cm}^{2}$ for detecting TGFs. The combination of XGRE and IDEE will give about $255 \mathrm{~cm}^{2}$ effective area for detecting electrons associated to TGFs. With a count rate capability of about 10 million counts $\mathrm{s}^{-1}$, XGRE should suffer from far fewer dead time and pile-up issues during bright TGF events, which were detrimental for previous detectors. Thus XGRE should derive precise measurements of light curves and spectra, even for the shortest TGF.

Using Monte Carlo simulations of the TARANIS, RHESSI AGILE and Fermi spacecrafts, we could estimate the response of their detectors to electrons and positrons and provide a quantitative comparison between them. By combining this knowledge with an approximative world map of detectable TGF density and with MC-PEPTITA Monte Carlo simulations of TGF propagation in the atmosphere, we could build an accurate model of the TGF detection rates of RHESSI, AGILE and Fermi. It could be used to estimate that TARANIS should detect about $200 \mathrm{TGFs}^{-1}$ and 25 TEBs $\mathrm{yr}^{-1}$.

Code availability. The GEANT4 mass model, TARANIS satellite, with XGRE and IDEE instruments is still under development and is not publicly available. However, simulations in specific configurations can be requested from the corresponding author: contact David Sarria (david.sarria.89@gmail.com).

The GEANT3 mass model of the RHESSI detector and spacecraft can be requested from David Smith (dsmith@scipp.ucsc.edu)

The GEANT3 mass model of the AGILE detectors and spacecraft can be requested from Martino Marsaldi (martino.marisaldi@uib.no), Marcello Galli (marcello.galli@enea.it) and Francesco Longo (franzlongo1969@ gmail.com).

The GEANT4 GDML mass model of the Fermi-GBM BGO and $\mathrm{NaI}$ detection units are publicly available as part of the GRESS software (Kippen et al., 2007). For this work, an approximative Fermi spacecraft model was built, which roughly respects the mass distri- 
bution of the spacecraft known from literature (see Meegan et al., 2009, and references therein).

MC-PEPTITA simulations can be requested from David Sarria (david.sarria.89@gmail.com). The MC-PEPTITA programme was developed under a contract of Centre National d'Etudes Spatiales (CNES) and Direction Generale de l'Armement (DGA), whose permissions are required in order to get access to the source code.

Data availability. The data generated for this work can be requested from the corresponding author: contact David Sarria (david.sarria.89@gmail.com).

The response matrices of Fermi GBM detectors are publicly available using the gbmrspgen tool, the usage of which is documented in the following website https://fermi.gsfc.nasa.gov/ssc/ data/analysis/scitools/gbmrspgen.html. The response matrix of the RHESSI instrument in the TGF context was made publicly available by D. M. Smith in the following website: http://scipp.ucsc.edu/ $\sim$ dsmith/tgflib_public/. The effective area vs. energy against X-ray and gamma ray photons (in the TGF context) for AGILE-MCAL is taken from Marisaldi et al. (2015).

The V2.3.2014 gridded satellite lightning data were produced by the NASA LIS/OTD Science Team (Principal Investigator, Dr. Hugh J. Christian, NASA/Marshall Space Flight Centre) and are available from the Global Hydrology Resource Centre (https://ghrc. nsstc.nasa.gov/home/). 
Appendix A: Determining detectors' averaged effective areas

We use a custom method to determine the average effective area of an instrument for detecting TGFs (or TEBs). Using a simulation of a given instrument, we can launch monoenergetic beams of particles of energy $E$ and determine the number that has been detected $N_{\mathrm{d}}(E)$. We can then determine $S_{\mathrm{X}}^{\mathrm{Y}}(E)$, the effective area at the energy $E$, where $Y$ corresponds to the event type (TGF or TEB) and $X$ designates the detector (XGRE, IDEE, RHESSI, GBM or MCAL). Assuming there are $N_{\text {launch }}$ particles drawn uniformly from an area $S_{\text {launch }}$ (that should be higher than the area of the whole satellite):

$S_{\mathrm{X}}^{\mathrm{Y}}(E)=N_{\mathrm{d}}(E) \times \frac{S_{\text {launch }}}{N_{\text {launch }}}$.

$S_{\mathrm{X}}^{\mathrm{Y}}(E)$ can then be averaged over an assumed spectra of TGF (or TEB) to obtain a value $\sigma_{\mathrm{X}}^{\mathrm{Y}}$, characterising the average effective area of the detector for detecting a TGF (or TEB):

$\sigma_{\mathrm{X}}^{\mathrm{Y}}=\frac{\int_{20 \mathrm{keV}}^{20 \mathrm{MeV}} f_{Y}(E) S_{\mathrm{X}}(E) \mathrm{d} E}{\int_{20 \mathrm{keV}}^{20 \mathrm{MeV}} f_{Y}(E) \mathrm{d} E}$,

where $f_{Y}(E)$ is the assumed spectrum of the considered event type. We choose to use the photon and electron spectra at satellite altitude presented in Fig. 4 of Dwyer et al. (2008), assuming it is valid for all the orbits of previously mentioned experiments. We also assume that $10 \%$ of the electrons are actually positrons (as it is estimated from simulations of TEBs Sarria et al., 2015), and that the electron spectrum does not differ very much in its shape from the positron spectrum.
Appendix B: Uncertainties on the models used, and the impact of wrongly estimated effective areas and count thresholds of XGRE

RHESSI, Fermi-GBM and AGILE-MCAL models have been made for X- and gamma-ray detection, and such models are usually accurate at about $5 \%$. Concerning electron/positron detection, the RHESSI, Fermi and AGILE models have not been made for this purpose and no calibration measurements were taken for electrons (as far as we know); thus there is no easy way to know precisely what the accuracy of their response to electrons/positrons is and so to give an accuracy level for the effective areas presented in Fig. 2 and Table 1.

For TARANIS, using calibration measurement at detection unit level, the model was tested to be about $5 \%$ accurate against $\mathrm{X}$-rays, gamma rays and (low energy) electrons. The real value of effective areas may vary from the values presented after the extensive analysis of the results of the calibration measurements on board the satellite (planned in 2018), and it is impossible to predict exactly how. When all the calibration measurements are processed, the model should be accurate at less than $5 \%$ for X-ray, gamma-ray and electron detection. The response to positrons will be impossible to test against real measurements and will only rely on simulations.

Using the model presented in Sect. 4.4, it is possible to calculate the sensitivity of a X percent inaccuracy of the estimated TARANIS effective areas on the final TGF and TEB detection rate estimation. The results are presented in Table B1.

A count threshold value of $n_{\mathrm{T}}^{\min }=10$ (like in the main text) was assumed to obtain these estimations. Table B2 present how a change on $n_{\mathrm{T}}^{\min }$ can impact our final TGF/TEB detection rate estimations (according to the model described in Sect. 4.4). 
Table B1. Effect of an overestimated effective area $\left(\sigma_{\mathrm{T}}^{\mathrm{XXX}}\right)$ on the limit radius $\left(R_{\mathrm{T}}^{\lim }\right)$ and the TGF/TEB detection rate estimations $\left(N_{\mathrm{T}}^{\mathrm{XXX}, \mathrm{est}}\right)$.

\begin{tabular}{lcclcc}
\hline $\begin{array}{l}\text { TGF } \\
\sigma_{\mathrm{T}}^{\mathrm{TGF}} \text { (nadir) }\end{array}$ & $R_{\mathrm{T}}^{\lim }$ & $N_{\mathrm{T}}^{\mathrm{TGF}, \mathrm{est}}$ & $\begin{array}{l}\text { TEB } \\
\sigma_{\mathrm{T}}^{\mathrm{TEB}}\end{array}$ & $R_{\mathrm{T}}^{\lim }$ & $N_{\mathrm{T}}^{\mathrm{TEB}, \text { est }}$ \\
\hline $425 \mathrm{~cm}^{2}$ & $820 \mathrm{~km}$ & 202 & $255 \mathrm{~cm}^{2}$ & $72 \mathrm{~km}$ & 25 \\
$382 \mathrm{~cm}^{2}(10 \%$ error $)$ & $793 \mathrm{~km}$ & 195 & $230 \mathrm{~cm}^{2}(10 \%$ error $)$ & $70 \mathrm{~km}$ & 23.6 \\
$340 \mathrm{~cm}^{2}(20 \%$ error $)$ & $765 \mathrm{~km}$ & 185 & $204 \mathrm{~cm}^{2}(20 \%$ error $)$ & $68 \mathrm{~km}$ & 22.3 \\
\hline
\end{tabular}

Table B2. Effect of an underestimated count threshold $\left(n_{\mathrm{T}}^{\min }\right)$ on the limit radius $\left(R_{\mathrm{T}}^{\lim }\right)$ and the TGF/TEB detection rate estimations $\left(N_{\mathrm{T}}^{\mathrm{XXX}, \mathrm{est}}\right)$.

\begin{tabular}{cccccc}
\hline $\begin{array}{c}\text { TGF } \\
n_{\mathrm{T}}^{\text {min }}\end{array}$ & $R_{\mathrm{T}}^{\lim }$ & $N_{\mathrm{T}}^{\mathrm{TGF}, \mathrm{est}}$ & $\begin{array}{c}\text { TEB } \\
n_{\mathrm{T}}^{\min }\end{array}$ & $R_{\mathrm{T}}^{\lim }$ & $N_{\mathrm{T}}^{\mathrm{TEB}, \text { est }}$ \\
\hline 10 & $820 \mathrm{~km}$ & 202 & 10 & $72 \mathrm{~km}$ & 25 \\
12 & $775 \mathrm{~km}$ & 189 & 12 & $68 \mathrm{~km}$ & 22.3 \\
15 & $724 \mathrm{~km}$ & 170 & 15 & $64 \mathrm{~km}$ & 19.7 \\
20 & $663 \mathrm{~km}$ & 149 & 20 & $59 \mathrm{~km}$ & 17.6 \\
30 & $582 \mathrm{~km}$ & 130 & 30 & $51 \mathrm{~km}$ & 13.2 \\
\hline
\end{tabular}


Author contributions. David Sarria prepared most of the manuscript. David Sarria, Remi Chipaux, Jean-Pierre Baronick contributed to the GEANT4 model of the XGRE instruments and TARANIS satellite. David Sarria performed all GEANT4 simulations (TARANIS XGRE, IDEE with satellite and FermiGBM). Francois Lebrun, Pierre-Louis Blelly and Philippe Laurent provided a detailed review of the manuscript, important feedback on the XGRE instrument, and the instrument comparison. Remi Chipaux provided a review of the manuscript. Damien Pailot and Miles Lindsey-Clark provided important data used to validate the GEANT4 model of the XGRE instrument, as well as important feedback on the instrument description (Sect. 2.1). Jean-Andre Sauvaud and Pierre Devoto provided the GEANT4 model of IDEE as well as important data for validation. Jean-Andre Sauvaud, Pierre Devoto and Lubomir Prech contributed to the IDEE instrument part of the manuscript (Sect. 2.2). Lubomir Prech provided an important review of the IDEE instrument description. Philippe Laurent performed a GEANT3 simulation on the RHESSI and AGILE mass models, which were provided by David Smith (RHESSI), Martino Marsaldi, Marcello Galli and Francesco Longo (AGILE).

Competing interests. The authors declare that they have no conflict of interest.

Acknowledgements. We thanks David M. Smith for providing the mass model, the RHESSI spacecraft, and discussion about its response to electrons. We thank Micheal S. Briggs for his help in estimating of the Fermi-GBM response to electrons. We thank Martino Marisaldi, Francesco Longo and Marcello Galli for providing the mass model of the AGILE spacecraft, and their help in discussing the response of MCAL to electrons. This work was granted access to the HPC resources of CALMIP supercomputing centre under the allocation 2015-p1505. We thank the CNES (Centre National d'Etudes Spatiales) for its financial support. The work of Lubomir Prech was supported by the Czech Science Foundation contract $17-06065 \mathrm{~S}$. We would like to thank the two anonymous referees for their valuable comments and suggestions that helped to improve the quality of this work.

Edited by: Antti Makela

Reviewed by: two anonymous referees

\section{References}

Agostinelli, S., Allison, J., Amako, K. et al.: GEANT4: A simulation toolkit, Nucl. Instrum. Methods, A506, 250-303, https://doi.org/10.1016/S0168-9002(03)01368-8, 2003.

Allison, J., Amako, K., Apostolakis, J. et al.: Geant4 developments and applications, IEEE T. Nucl. Sci., 53, 270-278, https://doi.org/10.1109/TNS.2006.869826, 2006.

Briggs, M. S., Fishman, G. J., Connaughton, V., Bhat, P. N., Paciesas, W. S., Preece, R. D., Wilson-Hodge, C., Chaplin, V. L., Kippen, R. M., von Kienlin, A., Meegan, C. A., Bissaldi, E., Dwyer, J. R., Smith, D. M., Holzworth, R. H., Grove, J. E., and Chekhtman, A.: First results on terrestrial gamma ray flashes from the Fermi Gamma-ray Burst Monitor, J. Geophys. Res.-
Space, 115, A07323, https://doi.org/10.1029/2009JA015242, 2010.

Briggs, M. S., Connaughton, V., Wilson-Hodge, C., Preece, R. D., Fishman, G. J., Kippen, R. M., Bhat, P. N., Paciesas, W. S., Chaplin, V. L., Meegan, C. A., von Kienlin, A., Greiner, J., Dwyer, J. R., and Smith, D. M.: Electron-positron beams from terrestrial lightning observed with Fermi GBM, Geophys. Res. Lett., 38, L02808, https://doi.org/10.1029/2010GL046259, 2011.

Briggs, M. S., Xiong, S., Connaughton, V., Tierney, D., Fitzpatrick, G., Foley, S., Grove, J. E., Chekhtman, A., Gibby, M., Fishman, G. J., McBreen, S., Chaplin, V. L., Guiriec, S., Layden, E., Bhat, P. N., Hughes, M., Greiner, J., Kienlin, A., Kippen, R. M., Meegan, C. A., Paciesas, W. S., Preece, R. D., Wilson-Hodge, C., Holzworth, R. H., and Hutchins, M. L.: Terrestrial gamma-ray flashes in the Fermi era: Improved observations and analysis methods, J. Geophys. Res.-Space, 118, 38053830, https://doi.org/10.1002/jgra.50205, 2013.

Cecil, D. J., Buechler, D. E., and Blakeslee, R. J.: Gridded lightning climatology from TRMM-LIS and OTD: Dataset description, Atmos. Res., 135-136, 404-414, https://doi.org/10.1016/j.atmosres.2012.06.028, 2014.

Cocco, V., Longo, F., and Tavani, M.: Simulation of the AGILE gamma-ray imaging detector performance: Part II, Nucl. Instrum. Meth. A, 486, 623-638, https://doi.org/10.1016/S01689002(01)02160-X, 2002.

Cohen, M. B., Inan, U. S., Said, R. K., Briggs, M. S., Fishman, G. J., Connaughton, V., and Cummer, S. A.: A lightning discharge producing a beam of relativistic electrons into space, Geophys. Res. Lett., 37, 1944-8007, https://doi.org/10.1029/2010GL044481, 2010.

Dwyer, J. R., Grefenstette, B. W., and Smith, D. M.: High-energy electron beams launched into space by thunderstorms, Geophys. Res. Lett., 35, L02815, https://doi.org/10.1029/2007GL032430, 2008.

Dwyer, J. R., Smith, D. M., and Cummer, S. A.: Highenergy atmospheric physics: Terrestrial gamma-ray flashes and related phenomena, Space Sci. Res., 173, 133-196, https://doi.org/10.1007/s11214-012-9894-0, 2012.

Fishman, G. J., Bhat, P. N., Mallozzi, R., Horack, J. M., Koshut, T., Kouveliotou, C., Pendleton, G. N., Meegan, C. A., Wilson, R. B., Paciesas, W. S., Goodman, S. J., and Christian, H. J.: Discovery of Intense Gamma-Ray Flashes of Atmospheric Origin, Science, 264, 1313-1316, https://doi.org/10.1126/science.264.5163.1313, 1994.

Fitzpatrick, G., Cramer, E., McBreen, S., Briggs, M. S., Foley, S., Tierney, D., Chaplin, V. L., Connaughton, V., Stanbro, M., Xiong, S., Dwyer, J., Fishman, G. J., Roberts, O. J., and von Kienlin, A.: Compton scattering in terrestrial gamma-ray flashes detected with the Fermi gamma-ray burst monitor, Phys. Rev. D, 90, 043008, https://doi.org/10.1103/PhysRevD.90.043008, 2014.

Gjesteland, T., Østgaard, N., Connell, P. H., Stadsnes, J., and Fishman, G. J.: Effects of dead time losses on terrestrial gamma ray flash measurements with the Burst and Transient Source Experiment, J. Geophys. Res.-Space, 115, A00E21, https://doi.org/10.1029/2009JA014578, 2010.

Gjesteland, T., Østgaard, N., Collier, A. B., Carlson, B. E., Eyles, C., and Smith, D. M.: A new method reveals more TGFs in the RHESSI data, Geophys. Res. Lett., 39, L05102, https://doi.org/10.1029/2012GL050899, 2012. 
Grefenstette, B. W., Smith, D. M., Dwyer, J. R., and Fishman, G. J.: Time evolution of terrestrial gamma ray flashes, Geophys. Res. Lett., 35, L06802, https://doi.org/10.1029/2007GL032922, 2008.

Inan, U. S., Piddyachiy, D., Peter, W. B., Sauvaud, J. A., and Parrot, M.: DEMETER satellite observations of lightninginduced electron precipitation, Geophys. Res. Lett., 34, L07103, https://doi.org/10.1029/2006GL029238, 2007.

Kippen, R. M., Hoover, A. S., Wallace, M. S., Pendleton, G. N., Meegan, C. A., Fishman, G. J., Wilson-Hodge, C. A., Kouveliotou, C., Lichti, G. G., von Kienlin, A., Steinle, H., Diehl, R., Greiner, J., Preece, R. D., Connaughton, V., Briggs, M. S., Paciesas, W. S., and Bhat, P. N.: Instrument Response Modeling and Simulation for the GLAST Burst Monitor, in: The First GLAST Symposium, edited by: Ritz, S., Michelson, P., and Meegan, C. A., vol. 921 of American Institute of Physics Conference Series, American Institute of Physics (AIP), Stanford, California, USA, 590-591, https://doi.org/10.1063/1.2757466, 2007.

Labanti, C., Marisaldi, M., Fuschino, F., Galli, M., Argan, A., Bulgarelli, A., Di Cocco, G., Gianotti, F., Tavani, M., and Trifoglio, M.: Design and construction of the Mini-Calorimeter of the AGILE satellite, Nucl. Instrum. Methods Phys. Res. A, 598, 470-479, https://doi.org/10.1016/j.nima.2008.09.021, 2009.

Lefeuvre, F., Blanc, E., and Pinçon, J. L.: TARANIS - a satellite project dedicated to the physics of TLEs and TGFs, in: American Institute of Physics Conference Series, vol. 1118 of American Institute of Physics Conference Series, American Institute of Physics (AIP), Corte, France, 3-7, https://doi.org/10.1063/1.3137711, 2009.

Lewis, H. W.: A robust method for tropopause altitude identification using GPS radio occultation data, Geophys. Res. Lett., 36, L12808, https://doi.org/10.1029/2009GL039231, 2009.

Lin, R. P., Dennis, B. R., Hurford, G. J., Smith, D. M., Zehnder, A., Harvey, P. R., Curtis, D. W., Pankow, D., Turin, P., Bester, M., Csillaghy, A., Lewis, M., Madden, N., van Beek, H. F., Appleby, M., Raudorf, T., McTiernan, J., Ramaty, R., Schmahl, E., Schwartz, R., Krucker, S., Abiad, R., Quinn, T., Berg, P., Hashii, M., Sterling, R., Jackson, R., Pratt, R., Campbell, R. D., Malone, D., Landis, D., BarringtonLeigh, C. P., Slassi-Sennou, S., Cork, C., Clark, D., Amato, D., Orwig, L., Boyle, R., Banks, I. S., Shirey, K., Tolbert, A. K., Zarro, D., Snow, F., Thomsen, K., Henneck, R., McHedlishvili, A., Ming, P., Fivian, M., Jordan, J., Wanner, R., Crubb, J., Preble, J., Matranga, M., Benz, A., Hudson, H., Canfield, R. C., Holman, G. D., Crannell, C., Kosugi, T., Emslie, A. G., Vilmer, N., Brown, J. C., Johns-Krull, C., Aschwanden, M., Metcalf, T., and Conway, A.: The Reuven Ramaty HighEnergy Solar Spectroscopic Imager (RHESSI), Sol. Phys., 210, 3-32, https://doi.org/10.1023/A:1022428818870, 2002.

Longo, F., Cocco, V., and Tavani, M.: Simulation of the AGILE gamma-ray imaging detector performance: part I, Nucl. Instrum. Meth. A, 486, 610-622, https://doi.org/10.1016/S01689002(01)02159-3, 2002.

Mailyan, B. G., Briggs, M. S., Cramer, E. S., Fitzpatrick, G., Roberts, O. J., Stanbro, M., Connaughton, V., McBreen, S., Bhat, P. N., and Dwyer, J. R.: The spectroscopy of individual terrestrial gamma-ray flashes: Constraining the source properties, J. Geophys. Res.-Space, 121, 11346-11363, https://doi.org/10.1002/2016JA022702, 2016JA022702, 2016.
Marisaldi, M., Fuschino, F., Tavani, M., Dietrich, S., Price, C., Galli, M., Pittori, C., Verrecchia, F., Mereghetti, S., Cattaneo, P. W., Colafrancesco, S., Argan, A., Labanti, C., Longo, F., Del Monte, E., Barbiellini, G., Giuliani, A., Bulgarelli, A., Campana, R., Chen, A., Gianotti, F., Giommi, P., Lazzarotto, F., Morselli, A., Rapisarda, M., Rappoldi, A., Trifoglio, M., Trois, A., and Vercellone, S.: Properties of terrestrial gamma ray flashes detected by AGILE MCAL below $30 \mathrm{MeV}$, J. Geophys. Res.-Space, 119, 1337-1355, https://doi.org/10.1002/2013JA019301, 2014.

Marisaldi, M., Argan, A., Ursi, A., Gjesteland, T., Fuschino, F., Labanti, C., Galli, M., Tavani, M., Pittori, C., Verrecchia, F., D’Amico, F., Østgaard, N., Mereghetti, S., Campana, R., Cattaneo, P., Bulgarelli, A., Colafrancesco, S., Dietrich, S., Longo, F., Gianotti, F., Giommi, P., Rappoldi, A., Trifoglio, M., and Trois, A.: Enhanced detection of terrestrial gammaray flashes by AGILE, Geophys. Res. Lett., 42, 9481-9487, https://doi.org/10.1002/2015GL066100, 2015.

Marisaldi, M., Smith, D. M., Brandt, S., Briggs, M. S., BudtzJørgensen, C., Campana, R., Carlson, B. E., Celestin, S., Connaughton, V., Cummer, S. A., Dwyer, J. R., Fishman, G. J., Fullekrug, M., Fuschino, F., Gjesteland, T., Neubert, T., Østgaard, N., and Tavani, M.: High-energy radiation from thunderstorms and lightning with LOFT, ArXiv e-prints, 2015.

Meegan, C., Lichti, G., Bhat, P. N., Bissaldi, E., Briggs, M. S., Connaughton, V., Diehl, R., Fishman, G., Greiner, J., Hoover, A. S., van der Horst, A. J., von Kienlin, A., Kippen, R. M., Kouveliotou, C., McBreen, S., Paciesas, W. S., Preece, R., Steinle, H., Wallace, M. S., Wilson, R. B., and Wilson-Hodge, C.: The Fermi gamma-ray burst Monitor, Astrophys. J., 702, 791-804, https://doi.org/10.1088/0004-637X/702/1/791, 2009.

Neubert, T., Kuvvetli, I., Budtz-Jørgensen, C., Østgaard, N., Reglero, V., and Arnold, N.: The atmosphere-space interactions monitor (ASIM) for the international space station, in: Proceedings of the ILWS Workshop, edited by: Gopalswamy, N., and Bhattacharyya, A., Goa, India, 448-451, 2006.

Nisi, R. S., Østgaard, N., Gjesteland, T., and Collier, A. B.: An altitude and distance correction to the source fluence distribution of TGFs, J. Geophys. Res.-Space, 119, 8698-8704, https://doi.org/10.1002/2014JA019817, 2014.

Ostgaard, N., Gjesteland, T., Hansen, R. S., Collier, A. B., and Carlson, B.: The true fluence distribution of terrestrial gamma flashes at satellite altitude, J. Geophys. Res.-Space, 117, 21562202, https://doi.org/10.1029/2011JA017365, 2012.

Ostgaard, N., Albrecthsen, K. H., Gjesteland, T., and Collier, A.: A new population of terrestrial gamma-ray flashes in the RHESSI data, Geophys. Res. Lett., 42, 10937-10942, https://doi.org/10.1002/2015GL067064, 2015.

Sarria, D., Blelly, P.-L., and Forme, F.: MC-PEPTITA: a Monte Carlo model for photon, electron and positron tracking in terrestrial atmosphere, Application for a terrestrial gamma-ray flash, J. Geophys. Res.-Space, 120, 3970-3986, 2014JA020695, https://doi.org/10.1002/2014JA020695, 2015.

Sarria, D., Blelly, P.-L., Briggs, M. S., and Forme, F.: Studying the time histogram of a terrestrial electron beam detected from the opposite hemisphere of its associated TGF, J. Geophys. Res.Space, 121, 4698-4704, https://doi.org/10.1002/2015JA021881, 2016. 
Smith, D. M., Lin, R. P., Turin, P., Curtis, D. W., Primbsch, J. H., Campbell, R. D., Abiad, R., Schroeder, P., Cork, C. P., Hull, E. L., Landis, D. A., Madden, N. W., Malone, D., Pehl, R. H., Raudorf, T., Sangsingkeow, P., Boyle, R., Banks, I. S., Shirey, K., and Schwartz, R.: The RHESSI spectrometer, Sol. Phys., 210, 33-60, https://doi.org/10.1023/A:1022400716414, 2002.

Smith, D. M., Lopez, L. I., Lin, R. P., and Barrington-Leigh, C. P.: Terrestrial gamma-ray flashes observed up to $20 \mathrm{MeV}$, Science, 307, 1085-1088, https://doi.org/10.1126/science.1107466, 2005.

Smith, D. M., Grefenstette, B. W., Splitt, M., Lazarus, S. M., Rassoul, H. K., Coleman, L. M., Dwyer, J. R., Lay, E. H., Holzworth, R. H., Cohen, M. B., Said, R., Inan, U. S., Chronis, T. G., and Takahashi, Y.: The anomalous terrestrial gamma-ray flash of 17 January 2004, AGU Fall Meeting Abstracts, American Geophysical Union, American Geophysical Union Fall Metting, San Francisco, California, USA, p. A1040, 2006.
Surkov, V. V. and Hayakawa, M.: Underlying mechanisms of transient luminous events: a review, Ann. Geophys., 30, 1185-1212, https://doi.org/10.5194/angeo-30-1185-2012, 2012.

Tavani, M., Barbiellini, G., Argan, A. et al.: The AGILE Mission, Astron. Astrophys., 502, 995-1013, https://doi.org/10.1051/0004-6361/200810527, 2009.

Ursi, A., Guidorzi, C., Marisaldi, M., Sarria, D., and Frontera, F.: Terrestrial gamma-ray flashes in the BeppoSAX data archive, J. Atmos. Sol.-Terr. Phy., 156, 50-56, https://doi.org/10.1016/j.jastp.2017.02.014, 2017.

Voss, H. D., Imhof, W. L., Walt, M., Mobilia, J., Gaines, E. E., Reagan, J. B., Inan, U. S., Helliwell, R. A., Carpenter, D. L., and Katsufrakis, J. P.: Lightning-induced electron precipitation, Nature, 312, 740-742, https://doi.org/10.1038/312740a0, 1984. 